\title{
Floristic composition in headwaters areas of the Piauitinga river Sub-Basin in
}

\section{Sergipe, Brazil}

Composição florística em áreas de nascentes na Sub-Bacia Hidrográfica do Rio Piauitinga em

\author{
Sergipe, Brasil
}

Composición florística en áreas de manantiales en la subcuenca del río Piauitinga en Sergipe, Brasil

\author{
Crislaine Costa Calazans \\ ORCID: http://orcid.org/0000-0002-6100-0608 \\ Universidade Federal de Sergipe, Brasil \\ E-mail: cris.calazans@yahoo.com.br \\ Gilmara da Silva Freire \\ ORCID: http://orcid.org/0000-0001-8374-6876 \\ Universidade Federal de Sergipe, Brasil \\ E-mail: gilfreire21@hotmail.com \\ Ana Paula do Nascimento Prata \\ ORCID: http://orcid.org/0000-0001-7922-8355 \\ Universidade Federal de Alagoas, Brasil \\ E-mail: apprata@yahoo.com.br \\ Robério Anastácio Ferreira \\ ORCID: http://orcid.org/0000-0003-4436-583X \\ Universidade Federal de Sergipe, Brasil \\ E-mail: roberioaf@yahoo.com.br
}

\begin{abstract}
Despite the riparian forest importance, it is evident that areas compose sub-basins undergo constant changes, culminating in these ecosystems' degradation. Thus, the floristic composition of nine headwaters of the Piauitinga River Sub-Basin, located in Sergipe, Brazil, will be presented. A collection of botanical material was taken within a radius of $50 \mathrm{~m}$ from the water edge of each source. The taxonomic identification of the specimens was developed considering specialized literature, taxonomic keys, expert consultation, comparison with dissected perianth and queries to the Virtual Herbarium. There were 190 species of vascular plants distributed in 146 genera and 55 families. 188 species of the Angiosperms group with 144 genera and 51 botanical families and the pteridophytes are represented by two species, belonging to two genera and two families. It is recommended the use of ecological restoration models that contemplate such species in future projects to be relayed in this planning unit.
\end{abstract}

Keywords: Degraded areas; Preservation; Species list.

\section{Resumo}

Apesar da importância das matas ciliares, constata-se que estas áreas que compõem as sub-bacias hidrográficas sofrem constantes alterações nos seus usos e funções, culminando em situações de grande degradação desses ecossistemas. Assim, será apresentada a composição florística de nove nascentes da Sub-Bacia Hidrográfica do Rio Piauitinga, situadas em Estância, Sergipe, Brasil. A coleta de material botânico foi realizada em um raio de $50 \mathrm{~m}$ a partir do olho d'água de cada nascente. A identificação taxonômica foi realizada mediante o uso de literatura especializada, chaves taxonômicas, consulta a especialistas, comparação com exsicatas e consultas ao Reflora Herbário Virtual. Foram registradas 190 espécies de plantas vasculares distribuídas em 146 gêneros e 55 famílias, das quais 188 são angiospermas com 144 gêneros e 51 famílias botânicas e as Samambaias são representadas por duas espécies pertencentes a dois gêneros e duas famílias. Face a elevada diversidade encontrada nas nascentes estudadas no Rio Piauitinga, é possível recomendar o uso de modelos de restauração ecológica que contemplem tais espécies em futuros projetos a serem realizados nesta unidade de planejamento.

Palavras-chave: Áreas degradadas; Preservação; Lista de espécies.

\section{Resumen}

A pesar de la importancia de los bosques de ribera, parece que estas áreas que conforman las subcuencas hidrográficas sufren constantes cambios en sus usos y funciones, culminando en situaciones de gran degradación de estos ecosistemas. Así, se presentará la composición florística de nueve manantiales de la Subcuenca del Río Piauitinga, ubicada en Estância, Sergipe, Brasil. La recolección de material botánico se realizó en un radio de $50 \mathrm{~m}$ desde la 
fuente de agua de cada manantial. La identificación taxonómica se realizó utilizando literatura especializada, claves taxonómicas, consulta con especialistas, comparación con exsiccates y consultas con Reflora - Herbario Virtual. Se registraron un total de 190 especies de plantas vasculares, distribuidas en 146 géneros y 55 familias, de las cuales 188 son angiospermas con 144 géneros y 51 familias botánicas, los helechos están representados por dos especies pertenecientes a dos géneros y dos familias. Dada la alta diversidad encontrada en las fuentes estudiadas en el río Piauitinga, es posible recomendar el uso de modelos de restauración ecológica que incluyan tales especies en los provectos futuros a realizarse en esta unidad de planificación.

Palabras clave: Áreas degradadas; Preservación; Lista de especies.

\section{Introduction}

The riparian forests correspond to the typologies of vegetation located on the banks of watercourses, headwaters and reservoirs. They play important roles, such as: protecting the banks of watercourses against erosion and silting and providing an adequate environment for local fauna, among others. Despite its importance, the rate of deforestation along riverbanks is accelerated (Silva, Cruz, Gonçalves, \& Mesquita, 2016). The preservation of the gallery forests has great importance, since they increase the flow of rivers by storing more water in the micro-basins, besides contributing to the maintenance of their quality. Throughout Brazil, gallery forests vary widely, each region has a predominance of species that are adapted to local conditions (Oliveira, Guimarães, Souza, Lima, \& Ferreira, 2015).

Water resources in general, and especially rivers and streams, are ecosystems that are altered by anthropic activities (Souza \& Bulhões, 2015) and these forests are essential for the management and conservation of river basins.

In Brazil, the accelerated urbanization process evidences the need for watershed management and green areas recovery, which are crucial for groundwater recharge and maintenance of water quality and quantity (Tundisi et al., 2015).

However, it is deeply necessary to emphasize the importance of the preservation of headwaters which are points of the groundwater outcropping, giving rise to a water source, and, considering their relevance, targets of recovery projects in the Piauitinga Sub-Basin Hydrographic Region.

The presence of riparian forests along rivers, streams and dams serves as an obstacle to the free flow of runoff water, reducing its speed and allowing its infiltration into the soil for absorption by plants and for feeding underground aquifers (Silva et al., 2016).

This paper presents a list of species occurring in headwaters areas in the Piauitinga River Sub-Basin in the municipality of Estância and aimed to provide subsidies for riparian forest recovery programs in degradation in the region, considering the vegetative characteristics, the knowledge of the species and their ecological functions.

\section{Methodology}

The present study was carried out in nine sources of the Piauitinga River Sub-Basin, located in the municipality of Estancia, Sergipe, Brazil. The Piauitinga River rises in the municipality of Lagarto and flows into the Piauí River, in the municipality of Estancia, which is part of the Pauí River Basin. The Piauí River Basin is in the Center-South region of the State of Sergipe, between coordinates $10^{\circ} 34^{\prime} 10^{\prime \prime}$ and $10^{\circ} 45^{\prime} 12^{\prime \prime} \mathrm{S}$ and $37^{\circ} 22^{\prime} 20$ "and $37^{\circ} 34^{\prime} 22^{\prime \prime} \mathrm{W}$, composing an area of 418.20 $\mathrm{km} 2$ (Figure 1) and its perimeter is $121.22 \mathrm{~km}$ (Moreira, 2008). 
Figure 1. Location of sampling spots in spring areas of the Piauitinga River sub-basin and municipality of Estância, Sergipe, Brazil.

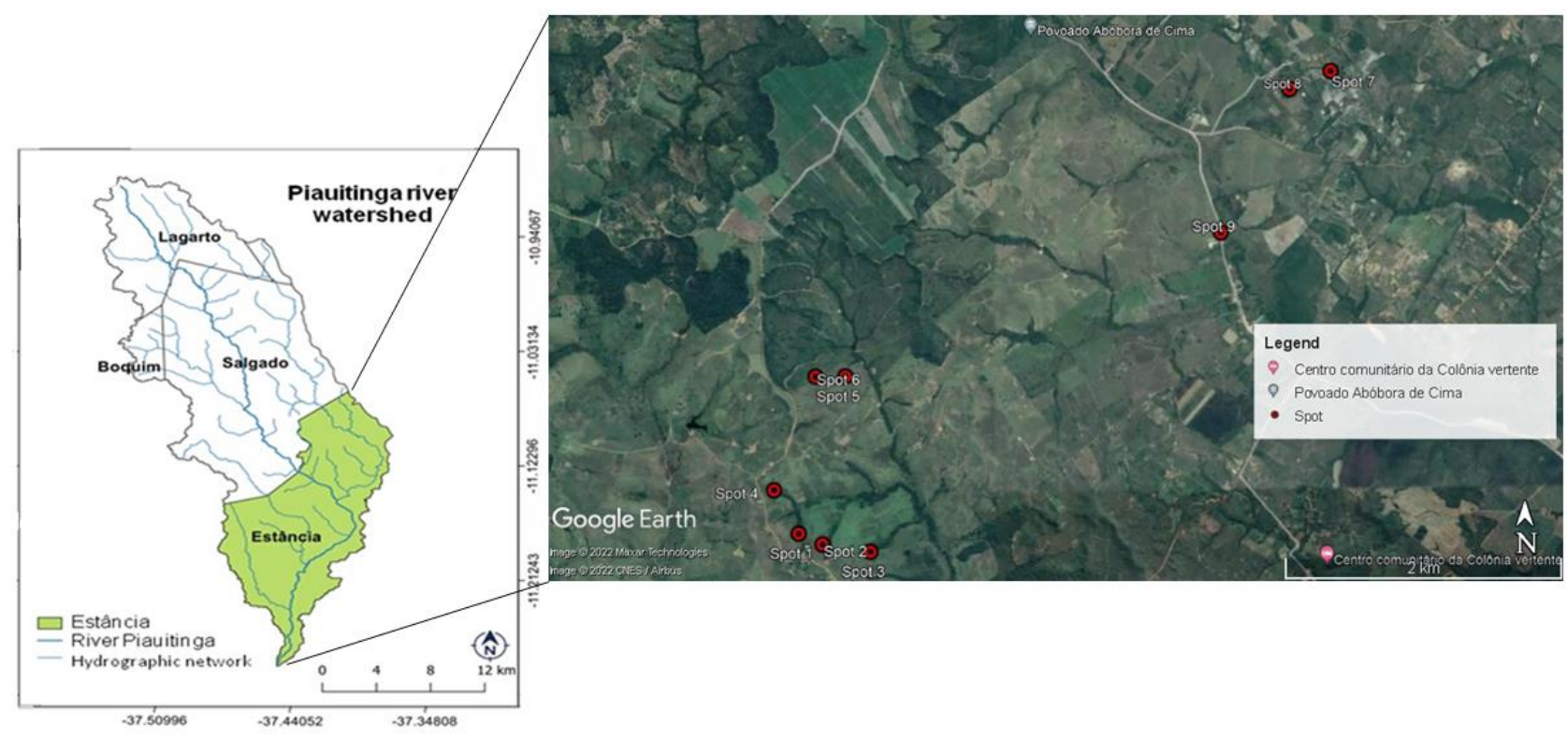

Source: Authors (2022).

The Piauitinga Sub-Basin is in the south-central part of the state of Sergipe, comprising the municipalities of Boquim, Estância, Lagarto, Salgado and Itaporanga D'Ájuda. The high course of the Piauitinga River receives contributions from tributaries with sufficient flow to be captured in Salgado, having great importance for water supply for human consumption, animal disintegration, irrigation, agriculture and livestock, element of industrial process, source of income for fishermen and its scenic beauty (Moreira, 2008).

The region vegetation was classified in the Submontane Semi-deciduous Seasonal Forest, transition between Estepic Savanna, Seasonal Forest and areas that suffered anthropization with cattle ranching and Arborized Estepic Savanna (Ministério do Meio Ambiente [MMA], 2021; Velloso, Rangel, \& Lima, 1991).

The climatic type of the region is the sub-humid, with rains distributed year-round, concentrating from April to August, with only one to three dry months. The mean annual precipitation is $1,396.8 \mathrm{~mm}$, with the highest average in the municipality of Estância (1,762 mm) and lowest in the municipality of Lagarto (1,064.8 mm) (Wanderley, 1998).

The soils of the region are: Red-Yellow Latosol, Red-Yellow Argisols and Flossic Neosol.

The floristic composition evaluations were carried out in nine headwaters, by means of collections of botanical material with reproductive structures, considering a radius of $50 \mathrm{~m}$ from the eye of each spring, determined with the aid of a set, totaling $7.85 \mathrm{~m} 2$. Considering the Permanent Preservation Area - APP the vegetation cover existing within a 50-m radius of the surroundings of a spring according to the Brazilian Forest Code (Law n. 12.651, 2012). The collection period lasted from October to December and from July to September, being carried out in dry and rainy periods of the region.

Samples of vascular plants (pteridophytes and phanerogams) were collected, belonging to different habits, such as: tree, shrub, grass and vine. At each headwater 3 to 5 samples, preferably fertile, were collected from each individual and the 
data of the botanical material were recorded: flower and fruit color, habit, collectors and geographical coordinates of the area.

Identification. All collected material was pressed and taken to oven drying at $60{ }^{\circ} \mathrm{C}$ for 48 to 72 hours. Later, they were duly deposited in the Herbarium of the Federal University of Sergipe (ASE) and the data were made available on Species link portal (Evironmental References and Information Center [CRIA], 2018). The taxonomic identification of the specimens was made by specialized literature, taxonomic keys, consultation with specialists, comparison with dissected perianth deposited in the general collection of the ASE Herbarium, queries to the Virtual Herbarium (REFLORA, 2018).

As for species classified, the Angiosperm Phylogeny Group VI classification system (The Angiosperm Phylogeny group [APG IV], 2016) was used and the spelling of the scientific names of the species and the determination of habit and origin of each species was performed according to the data available in Brazil Flora 2020 (The Brazil Flora [BFG], 2020). As for the habit, they were classified as herbaceous, shrub, arboreal and climbing. For the pteridophytes the list of species was presented in alphabetical order of botanical families, genera and species, including voucher, collector number and habit (Smith et al., 2006).

\section{Results and Discussion}

New records. A total of 190 species belonging to 53 families of vascular plants (Table 1) were found in the floristic surveys carried out in the plant formations of the Piauitinga River Sub-Basin, in the municipality of Estancia, Sergipe. Angiosperms contributed 188 species, 144 genera and 51 botanical families. The group of Pteridophytes was represented by two species belonging to two genera and two families. In terms of floristic richness, the Cyperaceae families with 26 species and Fabaceae with 24 species, followed by Asteraceae with 14 species and Poaceae with 12 species. These families accounted for $39.58 \%$ of total floristic wealth. The Malvaceae family obtained 10 taxa, followed by Rubiaceae with eight taxa, Melastomataceae with six taxa and Myrtaceae, Sapindaceae and Verbenaceae with five taxa each. 31 families were represented by only one specie.

Table 1. List of botanical families and species of Angiosperms and Pteridophytes from headwaters areas located at the Piauitinga River Sub-Basin, Estancia, Sergipe, Brazil, with their vouchers (ASE), collectors (name and number), and habits. Collector: Calazans, C.

\begin{tabular}{|c|c|c|c|}
\hline FAMILY/ESPECIES & VOUCHER & COLLECTOR & HABITAT \\
\hline \multicolumn{4}{|l|}{ AMARANTHACEAE } \\
\hline Alternanthera tenella Colla & 20913 & C 402 & Herbaceous \\
\hline \multicolumn{4}{|l|}{ ANACARDIACEAE } \\
\hline Anacardium occidentale $\mathrm{L}$. & 20987 & C 433 & Arboreal \\
\hline Mangifera indica $\mathrm{L}$. & 20985 & C. 432 & Arboreal \\
\hline Tapirira guianensis Aubl. & 20887 & C 508 & Arboreal \\
\hline Thyrsodium spruceanum Benth. & 20957 & C 446 & Arboreal \\
\hline \multicolumn{4}{|l|}{ ANNONACEAE } \\
\hline Duguetia sp. & 20974 & C 418 & Arboreal \\
\hline Xylopia frutescens Aubl. & 20886 & C 507 & Arboreal \\
\hline \multicolumn{4}{|l|}{ APOCYNACEAE } \\
\hline Himatanthus bracteatus (A.DC.) Woodson & 20897 & C 501 & Arboreal \\
\hline Mandevilla scabra (Hoffmanns. ex Roem. \& Schult.) K.Schum. & 20936 & C 379 & Climber \\
\hline Temnadenia odorifera (Vell.) J.F. Morales & 20925 & C 390 & Climber \\
\hline \multicolumn{4}{|l|}{ ASTERACEAE } \\
\hline Ageratum conyzoides $\mathrm{L}$. & 21633 & C 341 & Herbaceous \\
\hline
\end{tabular}


Research, Society and Development, v. 11, n. 2, e8311225428, 2022

(CC BY 4.0) | ISSN 2525-3409 | DOI: http://dx.doi.org/10.33448/rsd-v11i2.25428

\begin{tabular}{|c|c|c|c|}
\hline Albertinia brasiliensis Spreng. & 21637 & C 307 & Shrubby \\
\hline Centratherum punctatum Cass. & 20918 & C 397 & Herbaceous \\
\hline Conocliniopsis prasiifolia (DC.) R.M. King \& H. Rob. & 21588 & C 345 & Herbaceous \\
\hline Coreopsis lanceolata $\mathrm{L}$. & 20916 & C 399 & Herbaceous \\
\hline Synedrellan odiflora (L.) Gaertn. & 21593 & C 371 & Herbaceous \\
\hline Emilia sonchifolia (L.) DC. ex Wight & 20922 & C 393 & Herbaceous \\
\hline Lepidaploa cotoneaster (Willd. ex Spreng.) H.Rob. & 21590 & C 346 & Herbaceous \\
\hline Mikania cordifolia (L.f.) Willd. & 21594 & C 370 & Climber \\
\hline Moquiniastrum oligocephalum (Gardner) G. Sancho & 20966 & $\mathrm{C} 455$ & Herbaceous \\
\hline Pterocaulon virgatum (L.) DC. & 19372 & C 365 & Herbaceous \\
\hline Rolandra fruticosa (L.) Kuntze. & 21617 & C 368 & Shrubby \\
\hline Sphagneticola trilobata (L.) Pruski & 20937 & C 378 & Herbaceous \\
\hline Vernonanthura brasiliana (L.) H.Rob. & 21587 & C 324 & Shrubby \\
\hline \multicolumn{4}{|l|}{ BIGNONIACEAE } \\
\hline Handroanthus sp. & 20973 & C 417 & Arboreal \\
\hline Lundia corymbifera (Vahl) Sandwith & 21621 & C 308 & Climber \\
\hline \multicolumn{4}{|l|}{ BORAGINACEAE } \\
\hline Cordia sellowiana Cham. & 20952 & C 441 & Arboreal \\
\hline Heliotropium elongatum (Lehm.) I.M.Johnst. & 20910 & $\mathrm{C} 405$ & Herbaceous \\
\hline \multicolumn{4}{|l|}{ CARYOPLYLLACEAE } \\
\hline Spergula arvensis $\mathrm{L}$. & 20934 & C 381 & Herbaceous \\
\hline \multicolumn{4}{|l|}{ CELASTRACEAE } \\
\hline Maythenus sp. & 20964 & C 453 & Arboreal \\
\hline \multicolumn{4}{|l|}{ CHRYSOBALANACEAE } \\
\hline Hirtella racemosa Lam. & 20951 & C 440 & Arboreal \\
\hline Licania tomentosa (Benth.) Fritsch & 21601 & C 327 & Arboreal \\
\hline \multicolumn{4}{|l|}{ CONVOLVULACEAE } \\
\hline Ipomoea quamoclit $\mathrm{L}$. & 20924 & C 391 & Climber \\
\hline Jacquemontia bracteosa Meisn. & 20607 & C 374 & Climber \\
\hline \multicolumn{4}{|l|}{ CUCURBITACEAE } \\
\hline Momordica charantia $\mathrm{L}$. & 20915 & C 400 & Climber \\
\hline \multicolumn{4}{|l|}{ CYPERACEAE } \\
\hline Cyperus aggregatus (Willd.) Endl. & 21948 & C 21948 & Herbaceous \\
\hline Cyperus distans L. & 21972 & C 520 & Herbaceous \\
\hline Cyperus friburgensis Boeckeler & 21940 & C 21940 & Herbaceous \\
\hline Cyperus haspan $\mathrm{L}$. & 21956 & C 21956 & Herbaceous \\
\hline Cyperus laxus Lam. & 21963 & C 21963 & Herbaceous \\
\hline Cyperus luzulae (L.) Retz. & 21964 & C 21964 & Herbaceous \\
\hline Cyperus odoratus $\mathrm{L}$. & 21957 & C 21957 & Herbaceous \\
\hline Cyperus virens Michx. & 21961 & C 21961 & Herbaceous \\
\hline Cyperus sp. 1 & 21971 & C 521 & Herbaceous \\
\hline Cyperus sp. 2 & 21943 & C 21943 & Herbaceous \\
\hline Eleocharis geniculata (L.) Roem. \&Schult. & 21942 & C 21942 & Herbaceous \\
\hline Fimbristylis autumnalis (L.) Roem. \&Schult. & 21950 & C 21950 & Herbaceous \\
\hline Fimbristylis dichotoma (L.) Vahl & 21937 & C 21937 & Herbaceous \\
\hline Fuirena umbellate Rottb. & 21941 & C 21941 & Herbaceous \\
\hline Kyllinga brevifolia Rottb. & 21965 & C 21965 & Herbaceous \\
\hline Lipocarpha humboldtiana Nees & 21960 & C 21960 & Herbaceous \\
\hline
\end{tabular}


Research, Society and Development, v. 11, n. 2, e8311225428, 2022

(CC BY 4.0) | ISSN 2525-3409 | DOI: http://dx.doi.org/10.33448/rsd-v11i2.25428

\begin{tabular}{|c|c|c|c|}
\hline Lipocarpha micrantha (Vahl) G.C.Tucker & 21959 & C 21959 & Herbaceous \\
\hline Pycreus polystachyos (Rottb.) P.Beauv. & 21953 & C 21953 & Herbaceous \\
\hline Rhynchospora barbata (Vahl) Kunth & 21953 & C 21953 & Herbaceous \\
\hline Rhynchospora cephalotes (L.) Vahl & 21954 & C 21954 & Herbaceous \\
\hline Rhynchospora holoschoenoides (Rich.) Herter & 21962 & C 21962 & Herbaceous \\
\hline Rhynchospora nervosa (Vahl) Boeckeler & 21962 & C 21962 & Herbaceous \\
\hline Rhynchospora tenuis Link & 21952 & C 21952 & Herbaceous \\
\hline Scleria bracteata Cav. & 21955 & C 21955 & Herbaceous \\
\hline Scleria hirtella Sw. & 20942 & C 519 & Herbaceous \\
\hline Scleria gaertneri Raddi & 21939 & C 21939 & Herbaceous \\
\hline \multicolumn{4}{|l|}{ DILLENIACEAE } \\
\hline Curatella americana $\mathrm{L}$. & 20960 & C 449 & Arboreal \\
\hline Tetracera oblongata DC. & 21638 & C 301 & Shrubby \\
\hline \multicolumn{4}{|l|}{ EUPHORBIACEAE } \\
\hline Croton heliotropiifolius Kunth & 20953 & C 442 & Shrubby \\
\hline Croton sellowii Baill. & 20958 & $\mathrm{C} 447$ & Shrubby \\
\hline Microstachys corniculata (Vahl) Griseb. & 19370 & C 337 & Herbaceous \\
\hline \multicolumn{4}{|l|}{ FABACEAE } \\
\hline Andira fraxinifolia Benth. & 20888 & C 509 & Arboreal \\
\hline Bauhinia acuruana Moric. & 20969 & $\mathrm{C} 458$ & Shrubby \\
\hline Bowdichia virgilioides Kunth & 20892 & C 506 & Arboreal \\
\hline Camptosema sp. & 20935 & C 380 & Climber \\
\hline Chamae cristanictitans (L.) Moench. & 21632 & C 338 & Herbaceous \\
\hline Clitoria fairchildiana R.A.Howard & 20988 & $\mathrm{C} 434$ & Arboreal \\
\hline Desmodium barbatum (L.) Benth. & 20929 & C 386 & Shrubby \\
\hline Desmodium incanum (Sw.) DC. & 20927 & C 388 & Herbaceous \\
\hline Desmodium adscendens (Sw.) DC. & 21597 & C 367 & Herbaceous \\
\hline Dioclea lasiophylla Mart. ex Benth. & 21595 & C 355 & Climber \\
\hline Hymenaea rubriflora Ducke & 20968 & $\mathrm{C} 457$ & Arboreal \\
\hline Inga cayennensis Sagot ex Benth. & 20890 & C 511 & Arboreal \\
\hline Inga vera Willd. & 20983 & C 429 & Arboreal \\
\hline Lonchocarpus sericeus (Poir.) Kunth ex DC. & 20982 & $\mathrm{C} 428$ & Arboreal \\
\hline Mimosa pigra $\mathrm{L}$. & 21622 & C 307 & Shrubby \\
\hline Mimosa pudica $\mathrm{L}$. & 20919 & C 396 & Shrubby \\
\hline Mimosa tenuiflora (Willd.) Poir. & 20967 & C 456 & Shrubby \\
\hline Senna angulata (Vogel) H.S.Irwin \& Barneby & 19378 & C 328 & Shrubby \\
\hline Stryphnodendron pulcherrimum (Willd.) Hochr. & 20950 & C 439 & Arboreal \\
\hline Stylosanthes guianensis (Aubl.) Sw. & 21636 & C 361 & Herbaceous \\
\hline Swartzia apetala Raddi & 20975 & C 419 & Arboreal \\
\hline Swartzia flaemingii Raddi & 21599 & C 314 & Arboreal \\
\hline Tachigali densiflora (Benth.) L.G.Silva \& H.C.Lima & 20971 & $\mathrm{C} 420$ & Arboreal \\
\hline Tephrosia cinerea (L.) Pers. & 21624 & C 300 & Herbaceous \\
\hline \multicolumn{4}{|l|}{ GENTIANACEAE } \\
\hline Chelonanthus purpurascens (Aubl.) Struwe et al. & 19373 & C 357 & Herbaceous \\
\hline Coutoubeas picata Aubl. & 19380 & C 344 & Herbaceous \\
\hline \multicolumn{4}{|l|}{ HELICONIACEAE } \\
\hline Heliconia velloziana L.Emygd. & 20923 & C 392 & Herbaceous \\
\hline HUMIRIACEAE & & & \\
\hline
\end{tabular}


Sacoglottis guianensis Benth.

HYPERICACEAE

Vismia guianensis (Aubl.) Choisy

\section{LAMIACEAE}

Aegiphila integrifolia (Jacq.) Moldenke

Hyptis atrorubens Poit.

Hyptis sp.

Mesosphaerum pectinatum (L.) Kuntze

\section{LECYTHIDACEAE}

Eschweilera ovata (Cambess.) Mart. ex Miers

Lecythis pisonis Cambess.

LOGANIACEAE

Spigelia anthelmia $\mathrm{L}$.

\section{LYTHRACEAE}

Cuphea campestris Mart. ex Koehne

Cuphea racemosa (L.f.) Spreng

MALPIGHIACEAE

Byrsonima sericea DC.

Peixotoa parviflora A.Juss.

\section{MALVACEAE}

Eriotheca macrophylla (K.Schum.) A.Robyns

Gossypium hirsutum L.

Guazuma ulmifolia Lam.

Luehea ochrophylla Mart.

Pavonia cancellata (L.) Cav.

Pavonia rosa-campestris A.St.-Hil.

Sida sp.

Triumfetta semitriloba Jacq.

Urena lobata L.

Waltheria indica $\mathrm{L}$.

\section{MELASTOMATACEAE}

Clidemia capitellata (Bonpl.) D.Don

Miconia amoena Triana

Miconia ciliata (Rich.) DC.

Miconia minutiflora (Bonpl.) DC.

Pterolepis glomerata (Rottb.) Miq.

Pterolepis trichotoma (Rottb.) Cogn.

MORACEAE

Sp.

Sp.

\section{MUSACEAE}

Musa paradisiaca $\mathrm{L}$.

MYRTACEAE

Campomanesia aromatica (Aubl.) Griseb.

Eugenia punicifolia (Kunth) DC.

Myrcia loranthifolia (DC.) G.P.Burton \& E.Lucas

Myrcia sylvatica (G.Mey.) DC.

Myrciaria ferruginea O.Berg

\section{3}

20901

20899

21618

20921

20920

20948

20904

19374

20917

20939

20955

21627

20893

21592

20946

19383

20905

20933

21589

21630

19379

20928

20909

20902

20979

20965

21635

21631

21615

21614

20963

20895

20972

21623

21611

21604

\section{305}

C 414

Arboreal

416

C 323

C 394

C 395

C 437

C 411

C 350

Herbaceous

398

Herbaceous

C 335

Herbaceous

C 444

C 351

Arboreal

Shrubby

C 505

C 356

C 435

C 353

C 410

C 382

C 339

C 336

C 342

C 387

C 409

C 413

C 425

C 454

C 360

C 337

C 332

C 330

C 452

503

C 421

C 306

C 317

C 312
Arboreal

Arboreal

Shrubby

Arboreal

Shrubby

Arboreal

Shrubby

Climber

Shrubby

Herbaceous

Herbaceous

Herbaceous

Herbaceous

Shrubby

Shrubby

Shrubby

Shrubby

Shrubby

Shrubby

Arboreal

Shrubby

Arboreal

Shrubby
Arboreal 
Psidium guajava $\mathrm{L}$.

Psidium guineense $\mathrm{Sw}$.

Syzygium cumini (L.) Skeels

\section{OCHNACEAE}

Ouratea cuspidata Tiegh.

Sauvagesia erecta $\mathrm{L}$

\section{ONAGRACEAE}

Ludwigia octovalvis (Jacq.) P.H.Raven

\section{PASSIFLORACEAE}

Passiflora cincinnata Mast.

\section{PIPERACEAE}

Piper divaricatum G.Mey.

\section{POACEAE}

Andropogon bicornis $\mathrm{L}$.

Cenchrus echinatus L.

Cenchrus polystachios (L.) Morrone

Digitaria insularis (L.) Fedde

Megathyrsus maximus (Jacq.) B.K.Simon \& S.W.L.Jacobs

Panicum trichoides Sw.

Paspalum densum Poir.

Setaria parviflora (Poir.) Kerguélen

Steinchisma laxum (Sw.) Zuloaga

Taquara micrantha (Kunth) I.L.C.Oliveira \& R.P.Oliveira

Urochloa decumbens (Stapf) R.D.Webster

Urochloa plantaginea (Link) R.D.Webster

\section{POLYGALACEAE}

Polygala glochidata Kunth

POLYGONACEAE

Coccoloba laevis Casar.

Coccoloba rosea Meisn

\section{RUBIACEAE}

Alseis pickelii Pilg. \& Schmale

Borreria capitata (Ruiz \& Pav.) DC.

Borreria humifusa Mart.

Borreria verticillata (L.) G.Mey.

Eumachia chaenotricha (DC.) C.M. Taylor \& Razafim.

Genipa americana L.

Guettarda blanchetiana Müll.Arg

Tocoyena formosa (Cham. \& Schltdl.) K.Schum.

\section{RUTACEAE}

Zanthoxylum rhoifolium Lam.

Citrus $\times$ aurantium $\mathrm{L}$

\section{SALICACEAE}

Casearia sylvestris $\mathrm{Sw}$.

Casearia javitensis Kunth

\section{SAPINDACEAE}

Allophylus edulis (A.St.-Hil. et al.) Hieron. ex Niederl.

Cupania impressinervia Acev. - Rodr.

20896

20947

20900

20959

21591

20914

19375

21598

21969

21966

21968

21945

21967

21609

21944

20885

21947

21970

20945

20944

20907

21600

20970

21612

20906

21608

20912

20977

20949

20940

20962

20978

20976

20889

21610

19377

20986

$\begin{array}{ll}\text { C 502 } & \text { Arboreal } \\ \text { C 436 } & \text { Arboreal } \\ \text { C 415 } & \text { Arboreal }\end{array}$

C 448

Shrubby

C 358

Herbaceous

C 401

Herbaceous

Climber

Shrubby

C 369

C 21969

Herbaceous

C 21966

Herbaceous

C 21968

Herbaceous

C 21965

Herbaceous

C21967

Herbaceous

C 376

Herbaceous

C21944

Herbaceous

C 515

Herbaceous

C 21947

Herbaceous

C 21970

Herbaceous

C 516

Herbaceous

C 517

Herbaceous

C 408

Herbaceous

C 315

Shrubby

C 459

Shrubby

C 312

Arboreal

C 409

Herbaceous

C 375

Herbaceous

C 403

Herbaceous

C 423

Shrubby

C 438

Arboreal

C 323

C 451

Arboreal

Shrubby

C 424

Arboreal

C 422 Shrubby

510

Shrubby

C 316

Shrubby

$\begin{array}{ll}\text { C } 331 & \text { Arboreal } \\ \text { C 431 } & \text { Arboreal }\end{array}$




\begin{tabular}{|c|c|c|c|}
\hline Matayba guianensis Aubl. & 21605 & C 319 & Arboreal \\
\hline Paullinia trigonia Vell. & 21603 & C 303 & Climber \\
\hline Serjania salzmanniana Schltdl. & 21596 & C 366 & Climber \\
\hline \multicolumn{4}{|l|}{ SAPOTACEAE } \\
\hline Manilkara salzmannii (A.DC.) H.J.Lam & 20989 & C 460 & Arboreal \\
\hline Micropholis gardneriana (A.DC.) Pierre & 21628 & C 333 & Arboreal \\
\hline \multicolumn{4}{|l|}{ SIMAROUBACEAE } \\
\hline Sp. & 21634 & C 318 & \\
\hline \multicolumn{4}{|l|}{ SOLANACEAE } \\
\hline Cestrum axillare Vell. & 20894 & C 504 & Arboreal \\
\hline Solanum agrarium Sendtn. & 20911 & C 404 & Shrubby \\
\hline Solanum paniculatum $\mathrm{L}$. & 20954 & C 443 & Shrubby \\
\hline Solanum stipulaceum Willd. Ex Roem. \& Schult. & 21619 & C 322 & Shrubby \\
\hline \multicolumn{4}{|l|}{ TRIGONIACEAE } \\
\hline Trigonia nivea Cambess. & 21625 & C 302 & Climber \\
\hline \multicolumn{4}{|l|}{ URTICACEAE } \\
\hline Cecropia pachystachya Trécul & 20961 & C 450 & Arboreal \\
\hline \multicolumn{4}{|l|}{ VERBENACEAE } \\
\hline Lantana camara $\mathrm{L}$. & 20908 & C 407 & Shrubby \\
\hline Stachytarpheta angustifolia (Mill.) Vahl & 19382 & C 352 & Herbaceous \\
\hline Stachytarpheta cayennensis (Rich.) Vahl & 20891 & C 512 & Herbaceous \\
\hline Stachytarpheta sp. & 20884 & C 513 & Herbaceous \\
\hline Stachytarpheta sp. & 28080 & C $205 \mathrm{~A}$ & Herbaceous \\
\hline \multicolumn{4}{|l|}{ VIOLACEAE } \\
\hline Pombalia calceolaria (L.) Paula-Souza & 21606 & C 362 & Herbaceous \\
\hline \multicolumn{4}{|l|}{ VITACAE } \\
\hline Cissus erosa Rich. & 21620 & C 310 & Climber \\
\hline \multicolumn{4}{|l|}{ VOCHYSIACEAE } \\
\hline Vochysia lucida C.Presl & 20898 & C 500 & Arboreal \\
\hline \multicolumn{4}{|l|}{ XYRIDACEAE } \\
\hline Xyris macrocephala Vahl & 20943 & C 518 & Herbaceous \\
\hline \multicolumn{4}{|l|}{ PTERIDOPHYTES } \\
\hline \multicolumn{4}{|l|}{ LYGODIACEAE } \\
\hline Lygodium venustum $\mathrm{Sw}$. & 19376 & C 347 & Herbaceous \\
\hline \multicolumn{4}{|l|}{ THELYPTERIDACEAE } \\
\hline Cyclosorusin terruptus (Willd.) H. Ito & 21602 & C 363 & Herbaceous \\
\hline
\end{tabular}

Source: Authors (2022).

The most representative genera were: Cyperus (Cyperaceae) with 10 species, followed by Rhynchospora (Cyperaceae) with 5 species, Stachytarpheta (Verbenaceae) with 4 species and by Borreria (Rubiaceae) Scleria (Cyperaceae), Solanum (Solanaceae), Desmodium, Mimosa (Fabaceae) and Miconia (Melastomataceae) with 3 species each. 141 genera were represented by one or two species.

The species Lygodium venuustum Sw. (Lygodiaceae) and Cyclosorus interruptus (Willd.) H. Ito (Thelipteridaceae) are the only representative of the pteridophytes group.

Considering exclusively the shrub-tree flora of the 90 species, the richest families were Fabaceae (17 species) and Myrtaceae (eight species). 
The families Cyperaceae and Poaceae were the most representative of the herbaceous strata, with 26 species and 12 species, respectively. This event may be related to the fact that the areas are clearly degraded. Individuals of these species are commonly found in areas with similar conservation conditions to the site studied. All the studied spots areas were classified as to previous land use as: extensive livestock farming or permanent crops (Calazans et al., 2020).

Figure 2. Botanic families most representative of the source areas of the Piauitinga River Sub-Basin, Estância, SE, Brazil. A. Byrsonima sericea Dc (Malpighiaceae); B. Centratherum punctatum Cass. (Asteraceae); C. Croton heliotropiifolius Kunth (Euphorbiaceae); D. Guazuma ulmifolia Lam (Malvaceae); E. Momordica charantia L. (Cucurbitaceae); F. Ludwigia octovalvis (Jacq.) P. H. Raven. (Onagraceae).

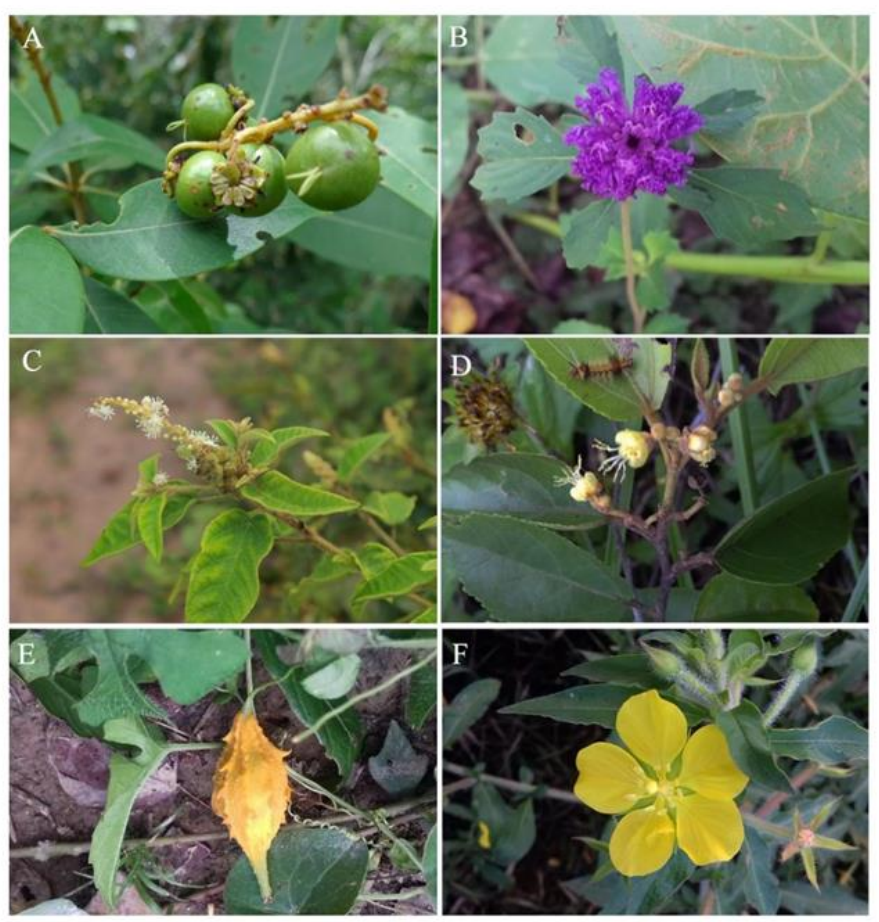

Source: Authors (2022).

The species Byrsonima sericea DC. (Malpighiaceae), Cupaniaim pressinervia Acev.-Rodr. (Sapindaceae), Eschweilera ovata Cambess Miers (Lecythidaceae), Himatanthus bracteatus (A.DC.) Woodson (Apocynaceae), Cecropia pachystachya Trécul (Urticaceae), Cyperus luzulae Hochst. Ex Steud. (Cyperaceae) and Lantana camara L. (Verbenaceae) stand out for the generalized occurrence, being specimens found in all areas of springs studied.

The most representative habit was the herbaceous ( $44.74 \%$ of the total), represented by 85 species distributed in 22 families, followed by arboreal (26.31\%) with 50 species belonging to 21 families. Among the shrubs, 40 species were recorded in 18 families $(21.05 \%)$, and the climbing plant habit was represented by 15 species $(7.89 \%)$.

These results confirm studies of plant diversity of the Atlantic Forest covering the arboreal and non-arboreal strata, the herbs and lianas are responsible for about $50 \%$ of the total wealth found (Pessoa \& Araujo, 2020).

The predominance of the herbaceous stratum was recorded in other floristic surveys in Atlantic Forest remnants (Almeida-Cortez, Tavares, Schulz, Pereira, \& Cierjacks, 2016; Oliveira et al., 2016; Santana, Rocha, Silva, Ribeiro, \& Prata, 2017). It is worth noting that the herbaceous species have great importance to kick off the ecological processes of restoration areas, due to the rapid cover and modifications promoted in the soils, both at chemical and physical aspects, by the deposition 
of organic matter and root growth, respectively.

Despite the advanced stage of degradation found in the study areas, the number of species recorded shows a high floristic diversity. Similar results were presented in floristic listings in Caatinga (Dry Forest) areas in Sergipe (Ferreira, Prata, \& Mello, 2013; Silva, Prata, \& Mello, 2013). The most representative families were Cyperaceae, Fabaceae, Asteraceae and Poaceae. The genera richest in number of species were: Cyperus and Rhynchospora belonging to the family Cyperaceae. For the tree species, there was a predominance of pioneer species. In this way, it is emphasized the importance of the understanding of species of different habits for the recovery and conservation of the environments, most notably the headwaters areas (Almeida-Cortez et al., 2016).

In a floristic composition study carried out in areas of springs in the same sub-basin in the municipality of Lagarto, 76 species from 58 genera were found, distributed in 36 botanical families (Ferreira et al., 2021).

The high number of individuals belonging to the Fabaceae family is also evidenced in work in which it reached a total of $24 \%$ of the study areas, this fact is associated with the strategies and adaptations of this family, due to the associations (Rhizobium and mycorrhiza), allowing the occurrence in the most diverse habitats.

Individuals of the Fabaceae family are widely used in projects to recover degraded areas since the species of this family can form a symbiosis with atmospheric nitrogen-fixing bacteria and mycorrhizal fungi. This technique can be considered low cost and with good results (Nogueira, Oliveira, Martins, \& Bernardes, 2012).

The number of species recorded shows a considerable floristic diversity, despite the advanced stage of degradation found in the study areas.

\section{Conclusion}

The most representative families were: Cyperaceae, Fabaceae, Asteraceae and Poaceae and the richest genera in species were Cyperus and Rhynchospora, belonging to the Cyperaceae family.

The fact that the herbaceous component predominates over the other vegetation habits may be closely related to the use and exportation historic of the riparian areas around the headwaters, since the predominance of shrub-tree species, in the case of riparian forests. since the riparian areas around the headwaters are in a degradation stage.

With this work development, it was possible to know and update the number of species cited for the municipality of Estancia and the collection of the ASE Herbarium, besides providing subsidies for future work on headwaters restoration at the Piauitinga River Sub-Basin.

\section{Acknowledgments}

We thank all the members of the "Adote um Manancial" Project for their help and support in the field research. We also thank the ASE Herbarium for the collaboration on the identification of all collected material. To the financing agencies, Secretariat of Environment and Water Resources of Sergipe state (SEMARH), CNPq, CAPES. Partnership: State Public Ministry - Lagarto - SE (MP-SE), Society for Multiple, Ecological and Arts Studies (Semear), Federal University of Sergipe / Department of Forestry Sciences (DCF / UFS).

\section{References}

Almeida-Cortez, J. S. de, Tavares, F. M., Schulz, K., Pereira, R. de C. A., \& Cierjacks, A. (2016). Floristic survey of the caatinga in areas with different grazing intensities, Pernambuco, Northeast Brazil. Journal of Environmental Analysis and Progress, 1(1), 43-51. https://doi.org/10.24221/jeap.1.1.2016.986.43-51

Calazans, C. C., Ferreira, R. A., da Silva Freire, G., Pereira, G. S., Mann, R. S., Souza, J. L., Torres, M. F. O., \& Nunes, V. V. (2020). Caracterização de 
Research, Society and Development, v. 11, n. 2, e8311225428, 2022

(CC BY 4.0) | ISSN 2525-3409 | DOI: http://dx.doi.org/10.33448/rsd-v11i2.25428

nascentes na sub-bacia hidrográfica do rio piauitinga, município de Estância, Sergipe (Characterization of springs in the sub-basin of the piauitinga river, municipality of Estância, Sergipe). Global science and technology, 13(1), 236-247. https://rv.ifgoiano.edu.br/periodicos/index.php/gst/article/view/1196

Centro de Referência em Informação Ambiental - (Evironmental References and Information Center). (2018). Species link. Retrieved August 18, 2020, from http://splink.cria.org.br/

Ferreira, E. V. R., Prata, A. P. D. N., \& Mello, A. A. de. (2013). Floristic list from a Caatinga remnant in Poço Verde, Sergipe, Brazil. Check List, 9(6), 1354. https://doi.org/10.15560/9.6.1354

Ferreira, R. A., Nascimento, M. I. C., Gama, D. C., Santos, T. I. S., Oliveira, D. G. de, Silva, A. J. da, \& Mello, A. A. de. (2021). Aspectos da vegetação e estado de conservação de nascentes do rio Piauitinga, Sergipe-Brasil (Aspects of vegetation and conservation status of Piauitinga river's springs, SergipeBrasil). Advances in Forestry Science, 8(4), 1591-1604. https://doi.org/10.34062/afs.v8i4.9699

Law no 12.651, May 25, 2012. Institute a new Brazilian floristic code. Oficial Journal of the Federative Republic of Brazil. Executive Department, Brasília, Federal District.

Ministério do Meio Ambiente. (2021). Mapas de cobertura vegetal dos biomas brasileiros (Maps of vegetation cover of the brazilian biomes). Federal Distict. Retrieved january 10, 2021, https://antigo.mma.gov.br/component/k2/item/7626-mapas-de-cobertura-vegetal.html

Moreira, F. D. (2008). Geotecnologia aplicada à sub-bacia hidrográfica do Rio Piauitinga e suas relações ambientais. (Geospatial technology applied to the Piauitinga River hydrographic sub-basin and its environmental relationship). Dissertation, Federal University of Sergipe, São Cristovão, Sergipe, Brazil.

Nogueira, N. O., Oliveira, O. M. de, Martins, C. A. da S., \& Bernardes, C. de O. (2012). Utilização de leguminosas para recuperação de áreas degradadas (Use of leguminosae for the recovery of degraded areas). Enciclopédia Biosfera, 8(21), 1-7.

Oliveira, E. V. da S., Gomes, L. A., Déda, R. M., Melo, L. M. S., Silva, A. C. da C., Farias, M. C. V., \& Prata, A. P. do N. (2016). Floristic survey of the Mata do Junco Wildlife Refuge, Capela, Sergipe State, Brazil. Hoehnea, 43(4), 645-667. https://doi.org/10.1590/2236-8906-28/2016

Oliveira, L. C. S., Guimarães, J. C. O., Souza, I. C. S., Lima, C. M., \& Ferreira, W. C. (2015). Levantamento florístico e fitossociológico da regeneração natural de uma mata de galeria localizada no município de Jataí - GO (Floristic and phytosociological survey of the natural regeneration of a gallery forest located in the municipality of Jataí - GO). Global Science and Technology, 8(3), 59-77. https://doi.org/10.14688/1984-3801/gst.v8n3p59-77

Pessoa, S. de V. A., \& Araujo, D. S. D. de. (2020). Tree species richness and composition in a fragmented landscape of the Brazilian lowland Atlantic Forest. Rodriguésia, 71. https://doi.org/10.1590/2175-7860202071003

Reflora. (2018). Virtual Herbarium. http://reflora.jbrj.gov.br/reflora/herbarioVirtual

Santana, J. P., Rocha, P. A. da, Silva, T. R. da, Ribeiro, A. de S., \& Prata, A. P. do N. (2017). Floristic characterization of Ibura national forest, Sergipe, Brazil. Bioscience Journal, 447-464. https://doi.org/10.14393/BJ-v33n2-33836

Silva, A. C. D. C., Prata, A. P. D. N., \& Mello, A. A. de. (2013). Flowering plants of the Grota do Angico Natural Monument, Caatinga of Sergipe, Brazil. Check List, 9(4), 733. https://doi.org/10.15560/9.4.733

Silva, G. A., Cruz, L. S., Gonçalves, F. D. S., \& Mesquita, J. . (2016). Crescimento da aroeira-vermelha no reflorestamento da mata ciliar. (Aroeira-vermelha growthon reparian forestre forestation). Agroforestalis News, 1(1), 8-12.

Smith, A. R., Pryer, K. M., Schuettpelz, E., Korall, E., Schneider, H. E., \& Wolf, P. G. (2006). A classification for extant ferns. Taxon, 55(3), $705-731$.

Souza, V. G., \& Bulhões, E. M. R. (2015). Environmental assessment through rapid assessment protocol low bed of the Paraíba do Sul River, In the Municipality of Campos Goytacazes / RJ. Cadegeo. http://www.cadegeo.uff.br/index.php/cadegeo/article/view/39

The Angiosperm Phylogeny group. (2016). An update of the Angiosperm Phylogeny Group classification for the orders and families of flowering plants: APG IV. Botanical Journal of the Linnean Society, 181(1), 1-20. https://doi.org/10.1111/boj.12385

The Brazil Flora. (2020). Brazilian Flora 2020: Innovation and collaboration to meet target 1 of the Global Strategy for Plant Conservation (GSPC). Rodriguésia, 69(4), 1513-1527. https://doi.org/10.1590/2175-7860201869402

Tundisi, J. G., Tucci, C. E. M., Spilki, F. R., Hespanhol, I., Cirilo, J. A., Scheuenstuhland, M. C. B., \& Periotto, N. A. (2015). Urban Waters in Brazil. In urban water challenges in the Americas: A perspective from the Academies of Sciences. (pp. 86-111). Rede Interamericana de Academias de Ciências (IANAS).

Velloso, H. P., Rangel, A. L. R., F., \& Lima, J. C. A. (1991). Classificação da vegetação brasileira adaptada a um sistema universal. (Brazilian vegetation classification adapted to a universal system).

Wanderley, L. L. (1998). Litoral Sul de Sergipe: uma proposta de proteção ambiental e desenvolvimento sustentável. (An environment protection proposition and sustainable development). Dissertation. Universidade Estadual Paulista Júlio de Mesquita Filho Rio Claro, São Paulo, Brazil. 